UCSBTH-94-11

hep-ph/9407322

\title{
COSMOLOGICAL CONSTRAINTS ON THE SCALE OF SUPERSYMMETRY BREAKING
}

\author{
RAGHAVAN RANGARAJAN* \\ Department of Physics \\ University of California \\ Santa Barbara, CA 93106
}

\begin{abstract}
We consider the cosmological and astrophysical constraints on the decay of massive $E_{8} \times E_{8}^{\prime}$ superstring axions associated with the hidden sector. We find that decay lifetimes greater than $1 \mathrm{~s}$ are ruled out by limits from nucleosynthesis, by limits on the distortion of the cosmic microwave and gamma ray backgrounds and by closure arguments. We conclude that $\Lambda>1.2 \times 10^{13} \mathrm{GeV}$, where $\Lambda$ is the scale of gaugino condensation in the hidden sector. This implies that the scale of supersymmetry breaking is greater than $10^{10} \mathrm{GeV}$. Significantly, our result agrees with the value of $5 \times 10^{13} \mathrm{GeV}$ for $\Lambda$ obtained independently by setting supersymmetric scalar masses equal to $m_{W}$.

(*: raghu@tpau.physics.ucsb.edu)
\end{abstract}

Nuclear Physics B 454 (1995) 357 
$E_{8} \times E_{8}^{\prime}$ superstring theories are anamoly free and hence very attractive [1,2]. Witten has shown that in such theories there is a model independent $U(1)$ Peccei-Quinn symmetry. When this symmetry breaks one gets an axion with a decay constant related to the compactification scale because the non-renormalizable interactions of the model independent axion arise as a result of compactification [3]. Furthermore, there are several model dependent axion degrees of freedom.

In $E_{8} \times E_{8}^{\prime}$ models compactified on a Calabi-Yau manifold the model independent axion degree of freedom corresponds to $B_{\mu \nu},(\mu, \nu=1, . ., 4)$, the zero mode of the antisymmetric tensor field $B_{M N}(M, N=1, . ., 10)$ which is essential for anamoly cancellation [3]. The model dependent axion is a linear combination of the zero modes $B_{m n}(m, n=5, . ., 10)$ [4]. Neither of the corresponding PQ symmetries survive compactification and the above axions have decay constants related to the compactification scale [5].

If $E_{8}^{\prime}$ breaks down to a non-abelian group there are two non-abelian groups today: $S U(3)_{C}$ and the non-abelian subgroup of $E_{8}^{\prime}$. The model independent and the model dependent axion degrees of freedom rearrange themselves to give two physical axions- the QCD axion $a$ and the $E_{8}^{\prime}$ axion $a^{\prime}$, with decay constants close to the compactification scale [6]. The energy density of the axions is proportional to $\Lambda^{\alpha} F_{a}^{\beta}(\alpha, \beta>0)$, where $\Lambda$ is the scale at which the underlying group gets strong. The QCD axion with $F_{a}>$ $10^{12} \mathrm{GeV}$ and $\Lambda \sim 200 \mathrm{MeV}$ will not have decayed by today and suffers an energy density problem. In this paper we study the cosmological and astrophysical constraints on the energy density of the $E_{8}^{\prime}$ axion and on its decay to photons to give limits on $\Lambda$ for the $E_{8}^{\prime}$ axion. We find that $\Lambda$ should be greater than $1.2 \times 10^{13} \mathrm{GeV} . \Lambda$ is also the scale of gaugino condensation in the hidden sector which breaks supersymmetry [7]. Since the masses of supersymmetric particles in the observed sector are approximately $10^{-1} \Lambda^{3} / M_{P l}^{2}$, this corresponds to supersymmetric particle masses greater than $1 \mathrm{GeV}$. The scale of supersymmetry breaking is given by $M_{S} \sim\left(\Lambda^{3} / M_{P l}\right)^{1 / 2}$. Thus, $M_{S}$ should be greater than $10^{10} \mathrm{GeV}$. Our result agrees with $\Lambda \simeq 5 \times 10^{13} \mathrm{GeV}$ obtained by setting $m_{\text {gaugino }} \simeq m_{W}[7]$. We find it significant that the result obtained by us using cosmological and astrophysical constraints agrees with the independent requirement from particle physics.

In a companion paper [8], we set $\Lambda$ equal to $5 \times 10^{13} \mathrm{GeV}$ and study the dilution of the baryon asymmetry due to axion decays. We find that for this value of $\Lambda$, most models of baryogenesis can not tolerate the large dilution (by a factor of $10^{7}$ ) of the baryon asymmetry.

World sheet instanton effects may add terms to the potential of the model dependent axion $[9,10]$ for most Calabi-Yau spaces (though not all). In such a case, the QCD axion will not get its mass purely from an $F \tilde{F}$ term but can have large contributions from other 
non-derivative couplings. The $E_{8}^{\prime}$ axion will be almost equivalent to $a_{1}$ below [11]. The mass of the $E_{8}^{\prime}$ axion will probably not be greatly affected. Hence, we ignore these effects below.

\section{II}

In an $E_{8} \times E_{8}^{\prime}$ superstring theory compactified on a Calabi-Yau manifold and in which the $E_{8}^{\prime}$ breaks down to a non-abelian group, the axion lagrangian in 4 dimensions is given by $[6]$

$$
\begin{aligned}
L=\frac{1}{2}\left(\partial_{\mu} a_{1}\right)^{2}+ & \frac{1}{2}\left(\partial_{\mu} a_{2}\right)^{2}+\frac{1}{32 \pi^{2} F_{1}} a_{1}\left(g^{2} F \tilde{F}+g^{\prime 2} F^{\prime} \tilde{F}^{\prime}\right) \\
& +\frac{1}{32 \pi^{2} F_{2}} a_{2}\left(g^{2} F \tilde{F}-g^{\prime 2} F^{\prime} \tilde{F}^{\prime}\right)
\end{aligned}
$$

where $a_{1}$ and $a_{2}$ are the model independent and model dependent axion degrees of freedom and $F_{1}$ and $F_{2}$ are the corresponding decay constants. Both $F_{1}$ and $F_{2}$ are related to the compactification scale and are about $10^{15} \mathrm{GeV}[11]$. $F$ can be taken to be either the electromagnetic or weak or strong interactions field strength tensor. $F^{\prime}$ corresponds to hidden sector gauge field strength tensors. The lagrangian can be rewritten as

$$
\begin{aligned}
L=\frac{1}{2}\left(\partial_{\mu} a\right)^{2} & +\frac{1}{2}\left(\partial_{\mu} a^{\prime}\right)^{2}+\frac{1}{32 \pi^{2} F_{a}} a\left(g^{2} F \tilde{F}\right) \\
& +\left(\frac{1}{32 \pi^{2} F_{a}^{\prime}} a^{\prime}\right)\left(g^{\prime 2} F^{\prime} \tilde{F}^{\prime}+g^{2} \frac{F_{2}^{2}-F_{1}^{2}}{F_{1}^{2}+F_{2}^{2}} F \tilde{F}\right)
\end{aligned}
$$

where

$$
\begin{gathered}
a=\frac{F_{1} a_{1}+F_{2} a_{2}}{\left(F_{1}^{2}+F_{2}^{2}\right)^{1 / 2}}, \\
a^{\prime}=\frac{F_{2} a_{1}-F_{1} a_{2}}{\left(F_{1}^{2}+F_{2}^{2}\right)^{1 / 2}}, \\
F_{a}=\frac{1}{2}\left(F_{1}^{2}+F_{2}^{2}\right)^{1 / 2},
\end{gathered}
$$

and

$$
F_{a}^{\prime}=F_{1} F_{2} /\left(F_{1}^{2}+F_{2}^{2}\right)^{1 / 2}
$$

$a$ is the QCD axion and $a^{\prime}$ is the $E_{8}^{\prime}$ axion. $a^{\prime}$ is massless at high temperatures. But it acquires a potential and a mass $m$ as the universe cools. The low temperature $(T<\Lambda)$ potential for $a^{\prime}$ is calculated to be $[6,12]$

$$
V=\frac{\pi}{4 M_{P l}^{2}} \Lambda^{6}\left|1-\exp \left(\mathrm{ia}^{\prime} / 15 \mathrm{~F}_{\mathrm{a}}^{\prime}\right)\right|^{2}
$$


Therefore the low temperature mass of the axion $a^{\prime}$ is

$$
m_{0}^{2}=\frac{\pi}{450} \frac{\Lambda^{6}}{M_{P l}^{2} F_{a}^{\prime 2}} .
$$

$a^{\prime}$ decays to two gluons, photons or $Z^{\prime}$ s or to a $W^{ \pm}$pair through the coupling $a^{\prime} F \tilde{F}$. The gluons, $W^{ \pm}$and $Z$ particles predominantly create jets of mesons and some baryons. At $T \sim \Lambda$, the non-abelian subgroup in the hidden sector gets strong leading to the hadronization of particles. The hidden sector hadrons will have masses of the order of $\Lambda$. Therefore we can ignore decays to hidden sector particles. A large fraction of the energy released as mesons is transferred to the photons, either through scattering or annihilations. We shall assume that only 20 per cent of the energy of the decaying axions is transferred to the photons i.e. $f=0.2$. This is an extremely conservative estimate.

The lifetime of the axion is given by

$$
\tau=8200 \pi^{5}\left(g_{1}^{4}+g_{2}^{4}+g_{3}^{4}\right)^{-1} \frac{F_{a}^{\prime 2}}{m_{0}^{3}}=5.2 \times 10^{-17} \mathrm{~s}\left(g_{1}^{4}+g_{2}^{4}+g_{3}^{4}\right)^{-1} \pi^{7 / 2} \frac{F_{a}^{\prime 5} M_{P l}^{3} \mathrm{GeV}}{\Lambda^{9}}
$$

where $g_{1,2,3}$ are the couplings of $U(1)_{Y}, S U(2)_{L}$ and $S U(3)$ respectively. We have set $\frac{F_{2}^{2}-F_{1}^{2}}{F_{1}^{2}+F_{2}^{2}}$ equal to 1 . We have checked that induced decays to photons and gluons due to coherent axion field oscillations $[13,14,15,16]$ are inhibited due to Hubble expansion. For $\tau$ between $1 \mathrm{~s}$ and about $10^{13} \mathrm{~s}$, the axion mass ranges between $10^{4} \mathrm{GeV}$ and about $10^{-1} \mathrm{GeV}$ and the axion decays predominantly to mesons through gluons. For this range of lifetimes one can ignore $g_{1}$ and $g_{2}$ and let $g_{3}=1$ (or $\alpha_{s} \sim 0.1$ ). For lifetimes greater than about $10^{13} \mathrm{~s}$ the axion mass is less than twice the pion mass and the axion can only decay to photons. If one rewrites eqn. (2a) in terms of $F_{E M} \tilde{F}_{E M}$, one gets the lifetime by replacing $\left(g_{1}^{4}+g_{2}^{4}+g_{3}^{4}\right)^{-1}$ in eqn. (5) by $\left(4 e^{4}\right)^{-1}$.

\section{III}

When the Peccei-Quinn symmetry breaks at early times the axion field need not be at the minimum of its potential. At a temperature $T_{i}$ the field will start to oscillate about the minimum of its potential with a period $m^{-1}(T)$. (Above $T_{i}$ the period of the oscillations is greater than the age of the universe $H^{-1}$. At high temperatures the mass has a temperature dependence, just as for the QCD axion.) We study the zero momentum mode of the $E_{8}^{\prime}$ axion field which can be treated as a condensate of zero momentum particles. (Higher momentum modes are redshifted.) The energy density of the axion field today is given by $[13,14,17]$

$$
\rho_{a_{0}}=m_{0} n_{0}=\frac{1}{2} m_{0}^{2} A_{0}^{2}
$$


$n_{0}$ is the number density today and $A_{0}$ is the amplitude of the oscillations today. A classical treatment is permitted as the number density is large [18]. The energy density can be rewritten in terms of parameters at $T_{i}$ when the field starts oscillating as

$$
\rho_{a_{0}}=m_{0} n_{i}\left(\frac{g_{s_{0}}}{g_{s_{i}}}\right) \frac{T_{0}^{3}}{T_{i}^{3}}=\frac{1}{2} m_{0} m_{i} A_{i}^{2}\left(\frac{g_{s_{0}}}{g_{s_{i}}}\right) \frac{T_{0}^{3}}{T_{i}^{3}} .
$$

$g_{s}$ is the effective number of relativistic degrees of freedom used to calculate the entropy. The above expression assumes that the axion field loses energy only due to the expansion of the universe and the variation of the mass with temperature and not due to decays. (The astrophysical constaints on massive decaying particles is given in terms of limits on the ratio of the energy density of the massive particles today, if they had not decayed, to the photon number density today.) $T_{i}$ is determined by the condition $m_{i}=3 H$ [13].

$$
T_{i}=\left[\frac{m_{i} M_{P l}}{5 g_{* i}^{1 / 2}}\right]^{1 / 2}
$$

$g_{*}$ is the effective number of relativistic degrees of freedom used to calculate the energy density. We shall take $g_{s_{i}}=g_{* i}$ and $g_{s_{f}}=3.9$. By the time the axion field starts oscillating, the axion mass has already attained its low temperature value in (4). One can verify this by checking that $m<3 H$ at $T=\Lambda$ when the field attains its low temperature mass (Appendix B). In deriving (8) we have assumed that the universe is radiation dominated when the axion field starts to oscillate. One can verify this by checking that $m>3 H$ at $T_{e q}$ when $\rho_{a}=\rho_{r}$, i.e., the axion field starts oscillating before the axion energy density dominates the universe (Appendix C). Combining (7) and (8) gives

$$
\rho_{a_{0}}=\frac{5^{3 / 2}}{2} \frac{g_{s_{0}}}{g_{* i}^{1 / 4}} m_{0}^{1 / 2} A_{i}^{2} \frac{T_{0}^{3}}{M_{P l}^{3 / 2}} .
$$

$A_{i}$ is taken to be $F_{a}^{\prime} \simeq 10^{15} \mathrm{GeV}$. In a non-inflationary universe, the spatial average of $A_{i} / F_{a}^{\prime}$ is $\pi / \sqrt{3}$. In an inflationary universe, setting $A_{i}$ equal to $F_{a}^{\prime}$ assumes that the universe lies in a typical post-inflationary region with $A_{i} / F_{a}^{\prime} \sim 1$. Linde has criticized this assumption. We refer the reader to Ref. [19] for a discussion of this issue. Since the number density of photons $n_{\gamma}=\left(1.2 / \pi^{2}\right) g_{\gamma} T^{3}$

$$
\rho_{a} /\left.n_{\gamma}\right|_{0}=0.62 \pi^{9 / 4} \frac{\Lambda^{3 / 2} F_{a}^{\prime 3 / 2}}{M_{P l}^{2}} .
$$

This is the ratio of the axion energy density today in the absence of decays to the photon number density today. 
Our method will be to look at the astrophysical and cosmological constraints that apply to $\rho_{a} /\left.n_{\gamma}\right|_{0}$ for different lifetimes $\tau$. For lifetimes between $1 \mathrm{~s}$ and $1.1 \times 10^{7} \mathrm{~s}$ we calculate the range of $\Lambda$ from (5) and substitute that in (10) and compare with the limits on the energy density from observations. We show that axions with lifetimes in this range are ruled out by cosmological arguments for $F_{a}^{\prime}=10^{15} \mathrm{GeV}$. Larger lifetimes are ruled out by limits on the distortions of the microwave and gamma ray backgrounds and by closure arguments. They would also give too small masses for the gauginos and supersymmetric scalars. $\tau$ less than $1 \mathrm{~s}$ corresponds to $\Lambda$ greater than $1.2 \times 10^{13} \mathrm{GeV}$.

\section{IV}

The presence of the axion has different effects during different epochs in the evolution of the universe. The presence of axions during the neutron-proton freeze-out $(\sim 1 \mathrm{~s})$ affects the ratio of neutrons to protons at freeze-out and thereby leads to a change in the amount of helium in the universe. Their presence during light element synthesis $(\sim 1$ minute) affects the amount of deuterium and ${ }^{3} \mathrm{He}$. The decay of axions to photons or to other particles that transfer their energy to photons increases $n_{\gamma}$ and dilutes $\eta$, the ratio of the baryon number density to the photon number density. This then alters the calculations of light element abundances. For $10^{4} \mathrm{~s}<\tau<10^{7} \mathrm{~s}$, the decay photons interact with the background thermal photons and create a cascade of photons with enough energy to photodissociate deuterium and helium. For lifetimes between $10^{5} \mathrm{~s}$ and (re)combination the decay photons change the shape of the spectrum of the cosmic background radiation. Decays occuring after recombination change the diffuse extra-galactic gamma ray spectrum. Decays of lifetimes greater than the age of the universe can lead to the axion energy density overclosing the universe.

We now explore the astrophysical constraints on the energy density of the axions for different lifetimes of the axion.

$\mathbf{1} \mathbf{s}<\tau<\mathbf{1 0}^{\mathbf{4}}$ s: Increased expansion rate and dilution of $\eta$

The interaction rate for the weak reactions $n+\nu_{e} \leftrightarrow p+e^{-}$and $p+\bar{\nu}_{e} \leftrightarrow n+e^{+}$ goes as $G_{F}^{2} T^{5}$ while the expansion rate of the universe is $\mathrm{H}=\left[(8 \pi / 3) \rho / \mathrm{M}_{\mathrm{Pl}}^{2}\right]^{(1 / 2)}$, where $\rho$ is the energy density of the universe [20]. When the weak reactions are faster than the expansion of the universe the ratio of the neutrons to protons is given by

$$
\frac{n}{p}=\exp \left[-\frac{\Delta m}{T}\right] .
$$

$\Delta m$ is the difference between the neutron and the proton mass. In the standard cosmology, at $t \sim 1 \mathrm{~s}(T \sim 1 \mathrm{MeV})$, the reaction rates become less than the expansion rate of the universe and the neutrinos freeze out and the protons and neutrons are no longer in 
chemical equilibrium. Thereafter the neutron-proton ratio does not change from its equilibrium value at the freeze-out temperature, except for free neutron decays. The precise temperature at which the freeze-out occurs is determined by the expansion rate of the universe which is influenced by the energy density of the axion. At slightly later times between 1 and 3 minutes $(0.3 \mathrm{MeV}>T>0.1 \mathrm{MeV})$, the light elements deuterium, ${ }^{3} \mathrm{He}$, ${ }^{4} \mathrm{He}$ and ${ }^{7} \mathrm{Li}$ are produced.

An increase in the expansion rate due to the axions causes the weak reactions above to freeze out at an earlier temperature. This leads to a a higher neutron to proton ratio and a higher percentage of ${ }^{4} \mathrm{He}$, if all the neutrons are converted into helium. However, the deuterium and ${ }^{3} \mathrm{He}$ abundances are sensitive to the expansion rate at temperatures between $0.08 \mathrm{MeV}$ and $0.04 \mathrm{MeV}$ when the strong reactions that convert deuterium and ${ }^{3} \mathrm{He}$ into ${ }^{4} \mathrm{He}$ freeze out. The amount of deuterium, ${ }^{3} \mathrm{He}$ and ${ }^{4} \mathrm{He}$ produced also depends on $\eta_{n s}$, the baryon-to-photon ratio during nucleosynthesis [21]. For a given $\eta_{0}$ today, the baryon-to-photon ratio before the axions decay is higher for higher axion energy density. The amount of ${ }^{4} \mathrm{He}$ today increases for higher $\eta_{n s}$ while the amount of deuterium and ${ }^{3} \mathrm{He}$ decreases because the rates of the reactions that convert deuterium and ${ }^{3} \mathrm{He}$ into ${ }^{4} \mathrm{He}$ are proportional to $\eta_{n s}$.

The constraint that ${ }^{4} \mathrm{He}$ makes up less than 25 percent of the energy density of the baryons in the universe and that the ratio of the abundances of deuterium and ${ }^{3} \mathrm{He}$ to the baryonic abundance is less than $1.0 \times 10^{-4}$ gives us limits on $\rho_{a} /\left.n_{\gamma}\right|_{0}$. For $1 \mathrm{~s}<\tau<10^{4} \mathrm{~s}$, the limit from light element abundances implies that $\rho_{a} /\left.n_{\gamma}\right|_{0}$ is less than $1.5 \times 10^{3} f^{-1} \mathrm{GeV}$ to $8.4 \times 10^{-5} f^{-1} \mathrm{GeV}$ [21]. But the axion energy density lies between $7.5 \times 10^{4} \mathrm{GeV}$ and $1.6 \times 10^{4} \mathrm{GeV}$. Therefore this range of lifetimes corresponding to $\Lambda$ between $1.2 \times 10^{13} \mathrm{GeV}$ and $4.3 \times 10^{12} \mathrm{GeV}$ and the axion mass between $1.2 \times 10^{4} \mathrm{GeV}$ and $5.5 \times 10^{2} \mathrm{GeV}$ is ruled out.

\section{$10^{4} \mathrm{~s}<\tau<1.1 \times 10^{7} \mathrm{~s}:$ Photodissociation}

For decays that occur in the time interval $10^{4} \mathrm{~s}<\tau<10^{7} \mathrm{~s}$, the decay photons can initiate electromagnetic cascades that can photodissociate deuterium and helium [22]. The high energy decay photons interact with the thermal photons producing electron-positron pairs. The electrons and positrons then lose their energy by inverse-Compton scattering the thermal photons to higher energies. These photons will repeat the above process till their energies fall below $E_{*} \simeq m_{e}^{2} / 22 T$, the threshhold for pair production. ( $m_{e}$ is the electron mass.) Photons with energy greater than $E_{*}$ and $Q$, the threshhold for photodissociation of an element, are more likely to pair produce than interact with the nuclei of the light elements [22]. The spectrum of the electromagnetic cascade initiated by a decay photon 
of energy $E\left(>E^{*}\right)$ is $[23]$

$$
\frac{d N^{E}}{d E_{\gamma}}= \begin{cases}\frac{24}{55} \sqrt{2} \frac{E}{\sqrt{E_{*}}} E_{\gamma}^{-3 / 2} & \text { for } 0 \leq E_{\gamma} \leq E_{*} / 2 \\ \frac{3}{55} E E_{*}^{3} E_{\gamma}^{-5} & \text { for } E_{*} / 2 \leq E_{\gamma} \leq E_{*} \\ 0 & \text { for } E_{*}<E_{\gamma}\end{cases}
$$

where the cascade spectrum has been normalized as

$$
\int_{0}^{E_{*}} E_{\gamma}\left(\frac{d N^{E}}{d E_{\gamma}}\right) d E_{\gamma}=E .
$$

The photodissociation of an element starts when $E_{*}$ becomes larger than the photodissociation threshhold for that element.

The mass fraction of an element is defined as $X_{k} \equiv n_{k} Z_{k} / n_{N}$, where $Z_{k}$ is the atomic number of the element, $n_{k}$ is the number density of the element and $n_{N}$ is the number density of the nucleons. The change in the mass fraction is given by $[22,23]$

$$
\begin{aligned}
\frac{d X_{k}}{d t}=-\frac{d n_{a}}{d t} \int_{0}^{\infty} \frac{d N}{d E} d E & {\left[\int_{Q_{k}}^{E_{*}(t)} \frac{d N^{E}}{d E_{\gamma}} \frac{X_{k} \sigma_{k}}{n_{e} \sigma_{C}} d E_{\gamma}\right.} \\
& \left.-\sum_{j \neq k} \int_{Q_{j}}^{E_{*}(t)} \frac{d N^{E}}{d E_{\gamma}} \frac{X_{j} \sigma_{j \rightarrow k}}{n_{e} \sigma_{C}} d E_{\gamma}\right]
\end{aligned}
$$

where $\sigma_{j \rightarrow k}$ is the partial cross-section for the dissociation of element $j$ to $k, \sigma_{k}$ is the total cross-section for photodissociation of element $k$ and $\sigma_{C}$ is the Compton scattering crosssection on the thermal electrons with density $n_{e}$. Anticipating the stringent conditions on the energy density of axions we assume above that $n_{e}$ is not changed significantly by the decays and cascade process. $\frac{d n_{a}}{d t}$ includes the change in the number density of axion due to decays only (and not due to the expansion of the universe).

$$
\frac{d n_{a}}{d t}=-\frac{n_{a}^{I}(t)}{\tau} \exp \left(-\frac{\mathrm{t}}{\tau}\right)
$$

where $n_{a}^{I}(t)$ is the value of $n_{a}$ at some very early time $t_{I}$ much less than $\tau$, scaled to the time of dissociation $t$. $e^{-t_{I} / \tau} \sim 1$. $\frac{d N}{d E}$ is the spectrum of decay photons. To simplify our calculation we only include photons coming directly from the decay of the axions. $\frac{d N}{d E}$ is then $2 \delta\left(E-m_{0} / 2\right)$. The ratio of the decay rates into photons and into gluons is $\alpha_{E M}^{2} / \alpha_{s}^{2} \sim 10^{-5}$. Therefore we include a factor of $f_{\gamma}=10^{-5}$ below. Including all the energy transferred to photons via all the axion decay channels can only tighten our constraints. 
Integrating (14) gives

$$
X_{k}^{f}-\left.X_{k}^{i} \simeq f_{\gamma} \frac{\rho_{a}}{n_{\gamma}}\right|_{0} \frac{8}{7 \eta_{0}}\left[-X_{k}^{i} \beta_{k}(\tau)+\sum_{j \neq k} X_{j}^{i} \beta_{j \rightarrow k}(\tau)\right]
$$

where

$$
\beta_{k}(\tau)=\frac{1}{\tau} \int_{t_{k}^{i}}^{\infty} \mathrm{dt} \exp \left(-\frac{\mathrm{t}}{\tau}\right) \int_{Q_{k}}^{E_{*}(t)} \frac{1}{E} \frac{d N^{E}}{d E_{\gamma}} \frac{\sigma_{k}\left(E_{\gamma}\right)}{\sigma_{C}\left(E_{\gamma}\right)} d E_{\gamma}
$$

and

$$
\beta_{j \rightarrow k}(\tau)=\frac{1}{\tau} \int_{t_{j}^{i}}^{\infty} \mathrm{dt} \exp \left(-\frac{t}{\tau}\right) \int_{Q_{j}}^{E_{*}(t)} \frac{1}{E} \frac{d N^{E}}{d E_{\gamma}} \frac{\sigma_{j \rightarrow k}\left(E_{\gamma}\right)}{\sigma_{C}\left(E_{\gamma}\right)} d E_{\gamma} .
$$

$i$ above represents the time when the photodissociation starts, i.e., when the cascade cutoff energy $E_{*}$ equals the photodissociation threshold $Q . \eta_{0}$ is the baryon-to-photon ratio today. Above we have used

$$
n_{a}^{I}(t) / n_{e}(t)=n_{a}^{I}\left(t_{0}\right) / n_{e}\left(t_{0}\right)
$$

and

$$
n_{e}\left(t_{0}\right) \simeq \frac{7}{8} n_{N_{0}}=\frac{7}{8} \eta_{0} n_{\gamma_{0}}
$$

The subscript 0 refers to quantities today and $N$ to nucleons.

$t^{i}$ is calculated from

$$
t^{i}=1.6 \times 10^{-25} g_{s}^{-1 / 2} \frac{M_{P l}^{7 / 4} \mathrm{GeV}}{m_{0}^{1 / 4} F_{a}^{\prime}\left(m_{e}^{2} / 22 Q\right)^{3 / 2}}
$$

where $Q$ is $2.2 \mathrm{MeV}$ for deuterium, $6.5 \mathrm{MeV}$ for ${ }^{3} \mathrm{He}$ and $20 \mathrm{MeV}$ for ${ }^{4} \mathrm{He}$ [24]. We have used the time-temperature relationship obtained in eqn.(11) of ref. [8] to obtain (19). $g_{s}$ is 3.9. The photodissociation of light elements only goes on till the energy of the decay photons itself falls below the threshhold for pair production. Then no cascade appears and photodissociation effects can be ignored. The time at which this occurs is calculated from

$$
E=\frac{m_{0}}{2}=\frac{m_{e}^{2}}{22 T}
$$

where

$$
m_{0}=1.2 \times 10^{-6}\left[\frac{F_{a}^{\prime 2} \mathrm{GeV}}{(\tau / \mathrm{s})}\right]^{1 / 3}
$$

(20b) is calculated from (5). Combining (20) with the $t-T$ relationship

$$
T=\left[\frac{\mathrm{s}}{t} \frac{1.6 \times 10^{-25}}{g_{s}^{1 / 2}} \frac{M_{P l}^{7 / 4} \mathrm{GeV}}{m_{0}^{1 / 4} F_{a}^{\prime}}\right]^{2 / 3}
$$


gives $t=1.1 \times 10^{7} \mathrm{~s}$. For decays that occur after this time, photodissociation of light elements is negligible.

For $10^{4} \mathrm{~s}<\tau<1.1 \times 10^{7} \mathrm{~s}$, the limits on $\rho_{a} /\left.n_{\gamma}\right|_{0}$ come from the constraints on the increase in deuterium and ${ }^{3} \mathrm{He}$ due to photodissociation of ${ }^{4} \mathrm{He}$. (The depletion of deuterium and ${ }^{3} \mathrm{He}$ is eclipsed by the increase in deuterium and ${ }^{3} \mathrm{He}$ due to photodissociation of ${ }^{4} \mathrm{He}$ as ${ }^{4} \mathrm{He}$ is $10^{4}$ times more abundant.)

$$
X^{f}\left(D+{ }^{3} \mathrm{He}\right)-\left.X^{i}\left(D+{ }^{3} \mathrm{He}\right) \simeq \frac{8}{7} Y^{i}\left({ }^{4} \mathrm{He}\right) \frac{\beta_{4} \mathrm{He}(\tau)}{\eta_{0}} r \frac{\rho_{a}}{n_{\gamma}}\right|_{0} f_{\gamma},
$$

where $r=\left(\frac{3}{4} \sigma_{\gamma^{4} \mathrm{He} \rightarrow n^{3} \mathrm{He}, p^{3} \mathrm{H}}+\frac{1}{2} \sigma_{\gamma^{4} \mathrm{He} \rightarrow n p D}\right) / \sigma_{\gamma^{4} \mathrm{He} \rightarrow \text { all }} \simeq 0.5$.

$\beta$ are computed numerically in Ref. [23] for a radiation dominated universe. The $t^{i}$ in our matter dominated universe are much earlier than those calculated in Ref. [23]. Hence $\beta_{4} \mathrm{He}$ will be greater in our case for lifetimes between $10^{4} \mathrm{~s}$ and $10^{7} \mathrm{~s}$. We take $\beta_{4} \mathrm{He}$ to be the peak value obtained in Ref. [23] of $1 \mathrm{GeV}^{-1}$. We take $Y^{i}\left({ }^{4} \mathrm{He}\right)<.25$ which implies $\eta<1.3 \times 10^{-9}$ and consequently $X^{i}\left(D+{ }^{3} \mathrm{He}\right)>3.4 \times 10^{-5}$. To be consistent with galactic chemical evolution $\left(D+{ }^{3} \mathrm{He}\right) / \mathrm{H}<10^{-4}$ or $X^{f}\left(D+{ }^{3} \mathrm{He}\right)<2.3 \times 10^{-4}$. Thus the upper limit on $\rho_{a} /\left.n_{\gamma}\right|_{0}$ is $1.0 \times 10^{-12} f_{\gamma}^{-1} \mathrm{GeV}$. But the calculated value of $\rho_{a} /\left.n_{\gamma}\right|_{0}$ is between $1.6 \times 10^{4} \mathrm{GeV}$ and $5.1 \times 10^{3} \mathrm{GeV}$. Hence the corresponding values of $\Lambda$ between $4.3 \times 10^{12} \mathrm{GeV}$ and $2.0 \times 10^{12} \mathrm{GeV}$ are ruled out. The corresponding values of the axion mass lie between $5.5 \times 10^{2} \mathrm{GeV}$ and $56 \mathrm{GeV}$.

$$
\tau>1.1 \times 10^{7} \mathrm{~s}:
$$

For lifetimes greater than $1.1 \times 10^{7} \mathrm{~s}$, the decay photons would distort the cosmic microwave background or show up in the gamma ray background spectrum. Such lifetimes would also gives supersymmetric masses less than $100 \mathrm{MeV}$. Lifetimes greater than the age of the universe would overclose the universe. Furthermore, if one assumes that the maximum baryon asymmetry $\eta \equiv n_{B} / n_{\gamma}$ produced in the early universe is $\mathrm{O}(1)[25,26]$ and that the baryon asymmetry today is $3 \times 10^{-10}$, then constraints on the dilution of the universe imply that $\Lambda$ must be greater than $7 \times 10^{12} \mathrm{GeV}[8]$.

\section{Conclusion:}

Thus we see that astrophysical and cosmological arguments imply that $\Lambda$ is greater than $1.2 \times 10^{13} \mathrm{GeV}$ or that $M_{S}$ is greater than $10^{10} \mathrm{GeV}$. Our result agrees with the value of $5 \times 10^{13} \mathrm{GeV}$ for $\Lambda$ obtained independently by setting supersymmetric scalar masses equal to $m_{W}$.

I would like to thank Mark Srednicki, Subir Sarkar, Robert Scherrer and Kiwoon Choi for very useful discussions. I would also like to thank the referee for pointing out the 
possibility of the induced decay of axions due to resonance effects. I am grateful to the Center for Particle Astrophysics at the University of California, Berkeley, where most of this work was completed, for their hospitality.

This work was supported by NSF Grant No. PHY91-16964. 


\section{Appendix A}

In this Appendix, we show that the universe is radiation dominated at the temperature $T \sim \Lambda$, when the axion attains it low temperature mass.

At $T=\Lambda$, the radiation energy density is given by

$$
\rho_{\text {rad }}=\frac{\pi^{2}}{30} g_{*} \Lambda^{4}
$$

while the axion energy density is given by

$$
\begin{aligned}
\rho_{a}=m_{0} n & =\frac{1}{2} m_{0}^{2} A^{2}(\Lambda) \\
& =\frac{\pi}{900} \Lambda^{4} \frac{\Lambda^{2}}{M_{P l}^{2}} \frac{A^{2}(\Lambda)}{F_{a}^{\prime 2}}
\end{aligned}
$$

The low temperature mass $m_{0}$ used above is given by (4). $g_{*}$ is 106.75 . At $T=\Lambda$ the field has not started oscillating (see Appendix B) and we assume that $A(\Lambda)$ is approximately $F_{a}^{\prime}$. Also, $\Lambda<M_{P l}$. Thus, $\rho_{a}<\rho_{\text {rad }}$ at $T=\Lambda$.

\section{Appendix B}

In this Appendix we show that the axion field starts oscillating at a temperature $T_{i}$ below $T=\Lambda$ at which the axion attains its low temperature mass. We shall show below that $m<3 H$ at $T=\Lambda$, i.e., the axion field has not yet started oscillating at $T=\Lambda$.

Since the universe is still radiation dominated at $T=\Lambda$ (see Appendix A),

$$
3 H=0.9 \pi^{3 / 2} g_{*}^{1 / 2} \frac{\Lambda^{2}}{M_{P l}}
$$

However,

$$
m_{0}=\left(\frac{\pi}{450}\right)^{1 / 2} \frac{\Lambda^{2}}{M_{P l}} \frac{\Lambda}{F_{a}^{\prime}}
$$

Since $\Lambda<F_{a}^{\prime}$ and $g_{*}=106.75, m<3 H$ at $T=\Lambda$. Thus the axion field starts oscillating at a temperature $T_{i}<\Lambda$, when $H$ has decreased sufficiently to satisfy $m=3 H$. 


\section{Appendix C}

In this Appendix we show that the universe is still radiation dominated at the temperature $T_{i}$ when the axion field starts oscillating. We actually show that the axion field is already oscillating by the time the temperature has decreased to $T_{\text {eq }}$, when the axion energy density is equal to the radiation energy density.

At $T=T_{\text {eq }}$,

$$
3 H=(12 \pi)^{1 / 2} \frac{A\left(T_{\mathrm{eq}}\right)}{M_{P l}} m_{0}
$$

Since $A\left(T_{\text {eq }}\right) \leq A\left(T_{i}\right)=F_{a}^{\prime}<0.2 M_{P l}, m>3 H$ at $T_{\text {eq. }}$. This implies that the universe started oscillating at a temperature greater than $T_{\mathrm{eq}}$, when the universe was still radiation dominated.

\section{REFERENCES}

[1] D. J. Gross, J. A. Harvey, E. Martinec and R. Rohm, Phys. Rev. Lett. 54 (1985) 502.

[2] M. B. Green and J. H. Schwarz, Phys. Lett. B149 (1984) 117.

[3] E. Witten, Phys. Lett. B149 (1984) 351.

[4] E. Witten, Phys. Lett. B155 (1985) 151.

[5] K. Choi and J. E. Kim, Phys. Lett. B154 (1985) 393.

[6] K. Choi and J. E. Kim, Phys. Lett. B165 (1985) 71.

[7] J. P. Derendinger, L. E. Ibanez and H. P. Nilles, Phys. Lett. B155 (1985) 65.

[8] R. Rangarajan, Nucl. Phys. B454 (1995) 369.

[9] X. G. Wen and E. Witten, Phys. Lett. B166 (1986) 397.

[10] M. Dine, N. Seiberg, X. G. Wen and E. Witten, Nucl. Phys. B289 (1987) 319.

[11] J. E. Kim, Phys. Rep. 150 (1987) 1.

[12] M.Dine, R. Rohm, N. Seiberg and E. Witten, Phys. Lett. B156 (1985) 55.

[13] J. Preskill, M. B. Wise, and F. Wilczek, Phys. Lett. B120 (1983) 127.

[14] L. Abbott and P. Sikivie, Phys. Lett. B120 (1983) 133.

[15] I. Tkachev, Sov. Astron. Lett. 12 (1986) 305.

[16] L. A. Kofman, A. D. Linde and A. A. Starobinsky, hep-th 9405187 (1994).

[17] M. Dine and W. Fischler, Phys. Lett. B120 (1983) 137. 
[18] A. D. Linde, Particle Physics and Inflationary Cosmology, (Harwood Academic Publishers, Switzerland, 1990).

[19] A. D. Linde, Phys. Lett. B201 (1988) 437; Phys. Lett. B259 (1991) 38.

[20] E. W. Kolb and M. S. Turner, The Early Universe (Addison-Wesley Publishing Company, 1990).

[21] R. J. Scherrer and M. S. Turner, Astrophys. J. 331 (1988) 19. The limits calculated in this paper are on $\rho / n_{\gamma}$ at $T=10^{12} K$. To obtain limits on $\rho_{a} /\left.n_{\gamma}\right|_{0}$ we multiply their results by a factor of $4 / 11$ to account for $e^{ \pm}$annihilation.

[22] D. Lindley, Astrophys. J. 294 (1985) 1.

[23] J. Ellis, G. B. Gelmini, J. L. Lopez, D. V. Nanopoulos and S. Sarkar, Nucl. Phys. B373 (1992) 399. This is a comprehensive paper on astrophysical constraints on massive decaying particles.

[24] J. Ellis, D. V. Nanopoulos and S. Sarkar, Nucl. Phys. B259 (1985) 175.

[25] I. Affleck and M. Dine, Nucl. Phys. B249 (1985) 361.

[26] A. D. Linde, Phys. Lett. B160 (1985) 243. 